\title{
Two Imported Cases of Babesiosis with Complication or Co-Infection with Lyme Disease in Republic of Korea
}

\author{
Hea Yoon Kwon', Jae Hyoung Im¹, Yun-Kyu Park², Areum Durey³, Jin-Soo Lee', Ji Hyeon Baek,** \\ ${ }^{1}$ Department of Internal Medicine, Inha University School of Medicine, Incheon 22212, Korea; ${ }^{2}$ Department of Emergency Medicine, Inha University \\ School of Medicine, Incheon 22212, Korea; ${ }^{3}$ Department of Tropical Medicine and Parasitology, Inha University School of Medicine, \\ Incheon 22212, Korea
}

\begin{abstract}
Babesiosis, caused by Babesia microti and B. divergens, is transmitted by lxodid ticks. Symptoms of babesiosis vary from a mild flu-like illness to acute, severe, and sometimes fatal and fulminant disease. In Korea, 7 imported babesiosis cases and 1 endemic case have been reported. We report 2 cases of severe babesiosis initially mistaken as malaria. The first patient was complicated by shock and splenic infarction, the other co-infected with Lyme disease. As the population traveling abroad increases every year, physicians should be aware of babesiosis which mimics malaria, co-infection with other diseases, and its complications.
\end{abstract}

Key words: Babesia microti, babesiosis, splenic infarction, co-infection, Lyme disease

\section{INTRODUCTION}

Babesiosis is a tick-mediated zoonosis caused by intraerythrocytic protozoa. The main species that cause disease in humans are Babesia microti and B. divergens, which are endemic to the northeastern and midwestern areas of the United States and Europe, respectively [1]. The symptoms of babesiosis are diverse, ranging from a subclinical flu-like illness to acute, severe disease occasionally resulting in fatality [2]. The patient usually presents with malaise, fever, fatigue, and headache. Hemolytic anemia and thrombocytopenia may be accompanied. If severe, organ dysfunction, mental change, pulmonary edema, renal failure, and splenic rupture can be present $[3,4]$.

Although not endemic, babesiosis in Korea has been continuously reported. Since the first imported case of babesiosis in Korea was reported in 1988, 11 cases of babesiosis have been reported until now [5-11]. Among them, only 1 case was endemic; the other cases were imported from Africa or the United States [11]. Although endemic babesiosis in Korea is not common, physicians should be aware of this disease because of increased travel to other countries and the increasing

- Received 13 August 2018, revised 8 October 2018, accepted 14 October 2018

*Corresponding author (jhbaek@inha.ac.kr)

(c) 2018, Korean Society for Parasitology and Tropical Medicine

This is an Open Access article distributed under the terms of the Creative Commons Attribution Non-Commercial License (http://creativecommons.org/licenses/by-nc/4.0) which permits unrestricted non-commercial use, distribution, and reproduction in any

medium, provided the original work is properly cited. incidence of babesiosis worldwide $[12,13]$. The symptoms and appearance of infected erythrocytes on blood smears are similar to erythrocytes infected by Plasmodium species. However, the treatment and course of babesiosis and malaria are different; therefore, awareness of the disease should be highlighted.

It is also noteworthy that babesiosis often occurs as a co-infection with other tick-borne diseases. Ixodes scapularis, the ticks which maintain B. microti, also transmit Borrelia burgdorferi and Anaplasma phagocytophilum [14]. Previous epidemiologic studies documented that 2/3 of patients with babesiosis experience concurrent Lyme disease $[14,15]$. Since co-infection with Lyme disease or anaplasmosis may lead to severe disease, suspicion of and early treatment for concurrent diseases are crucial when patients are in a fatal condition.

Here, we report 2 cases of babesiosis in Korea. Both cases were imported from the United States. They were first mistaken as malaria since malaria is endemic to the northern provinces of South Korea. Both patients presented severe symptoms and unusual complications; the first case was complicated by splenic infarction and the second case by co-infection with Lyme disease.

\section{CASE RECORD}

\section{Case 1}

A 50-year-old woman, a house-wife, visited our hospital complaining of fever, abdominal pain, and nausea. She had a histo- 

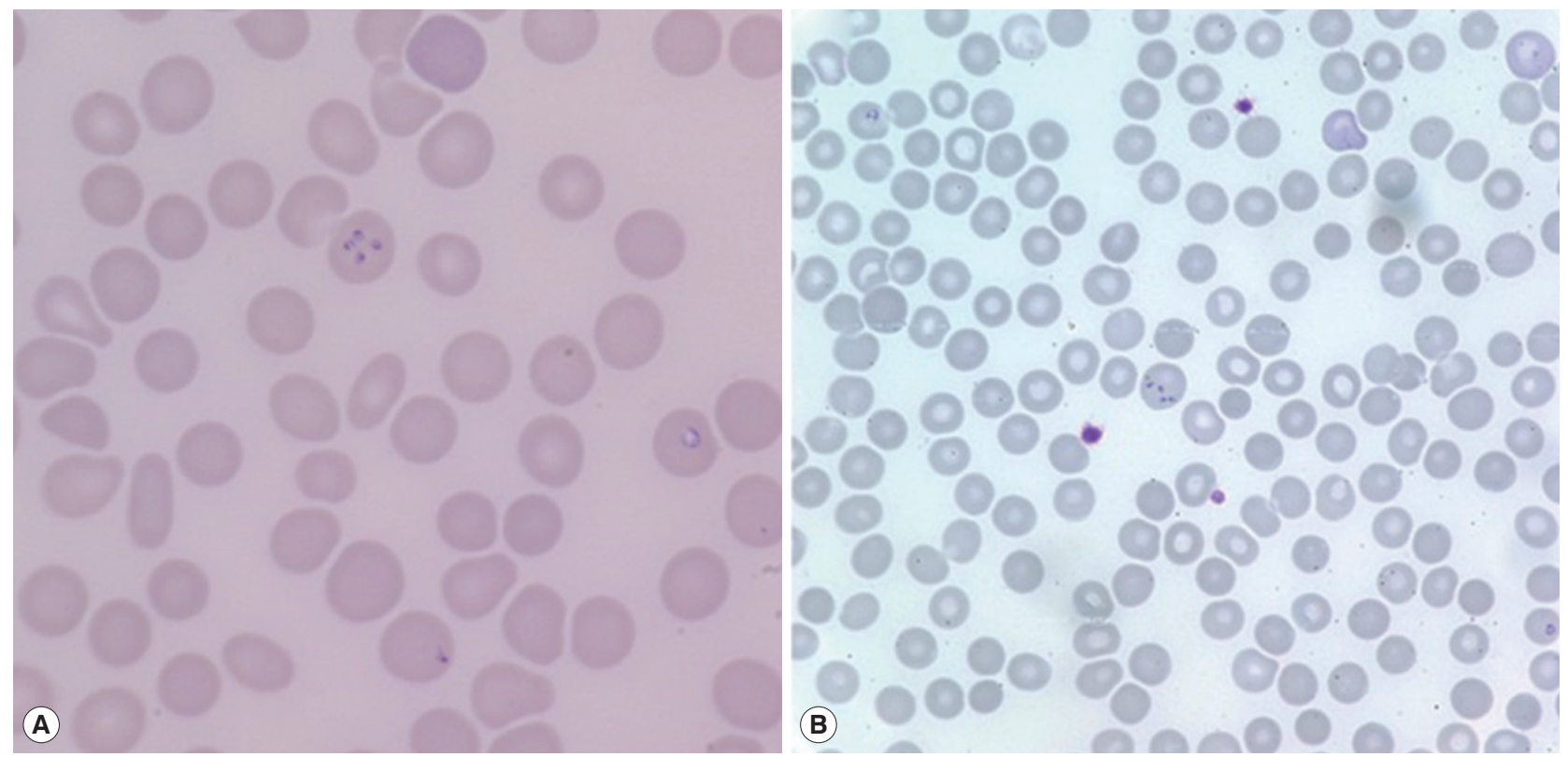

Fig. 1. Peripheral blood smears. Erythrocytes containing multiple ring-like trophozoites, cross-maltase form, are observed. Giemsa stained. (A) $\times 1,000$, (B) $\times 400$.
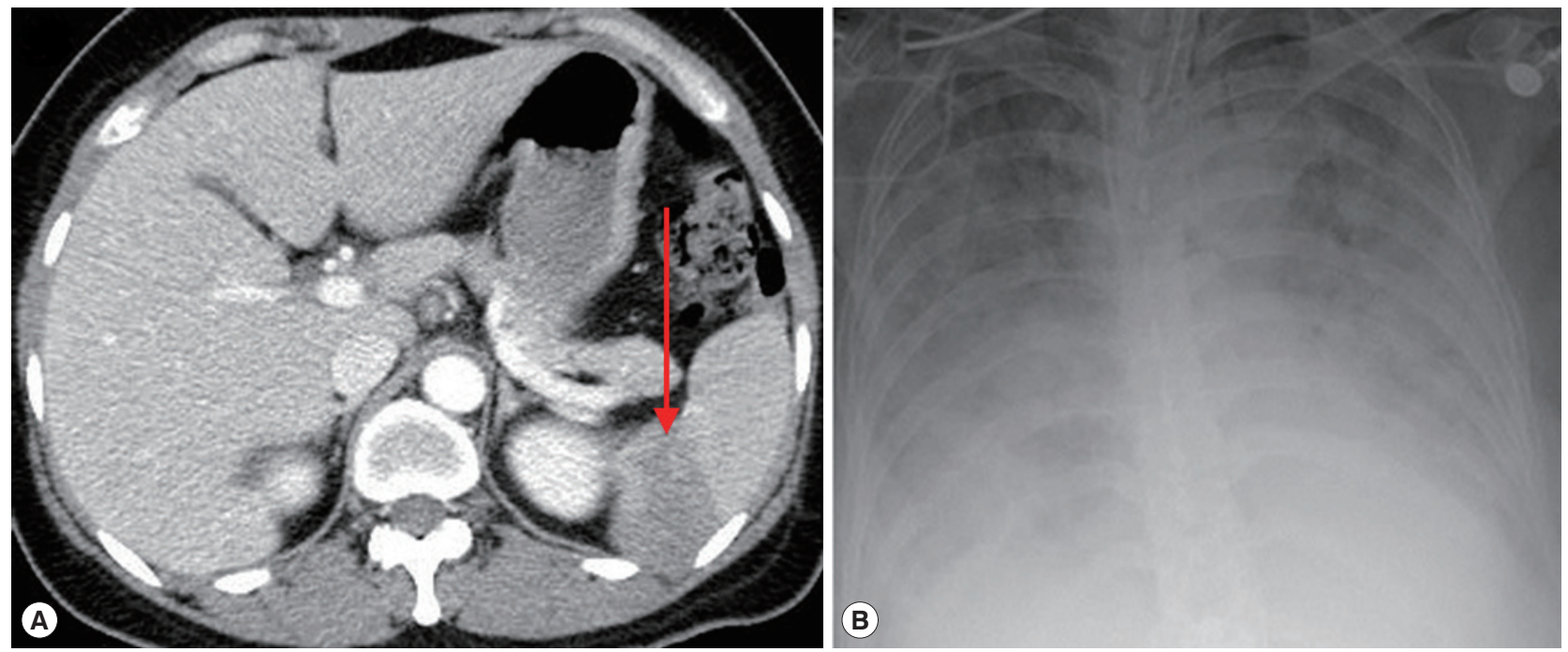

Fig. 2. Photographs of abdominal CT and chest X-ray. (A) Complications of severe babesiosis were demonstrated as splenic infarction. (B) Pulmonary congestion. An arrow indicates splenic infarction.

ry of suboccipital craniectomy due to right trigeminal nerve irritability 15 years ago. She also had right middle cerebral artery clipping because of a cerebral artery aneurysm rupture 2 years before.

She was residing in Incheon, South Korea. She visited New Jersey in the United States 4 weeks before the symptoms developed. In New Jersey, she went to a forest. Although she did not remember getting bitten by ticks or insects, she had an erythem- atous lesion on the right proximal calf area after visiting the forest, which was suspicious for a tick bite. Two days after detecting the skin lesion, headache, myalgia, fever, and chills developed.

On admission, her vital signs were: blood pressure 148/73 $\mathrm{mmHg}$, heart rate 120 beats per minute, respiratory rate 18 breaths per minute, and body temperature $40.7^{\circ} \mathrm{C}$. Laboratory examination showed white blood cells 5,610 cells/ $\mu$ (normal range 4,000-10,000 cells/ $/ \mathrm{l}$ ), hemoglobin $11.2 \mathrm{~g} / \mathrm{dl}$ (normal 
A

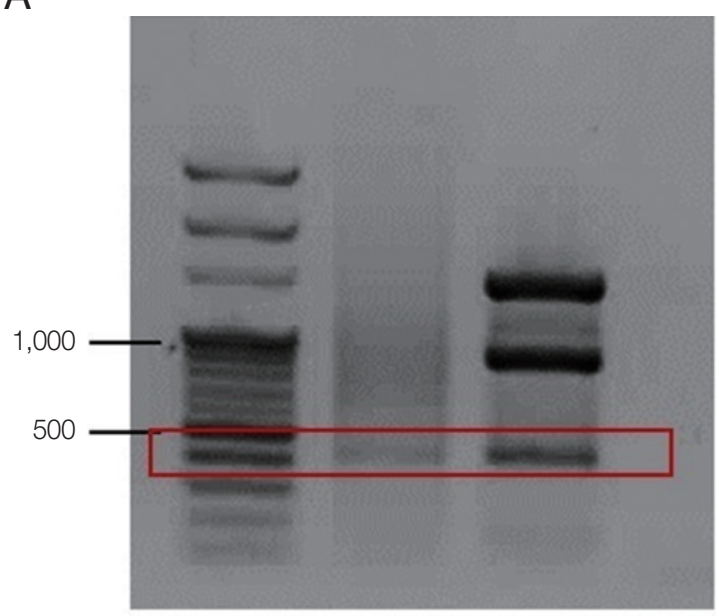

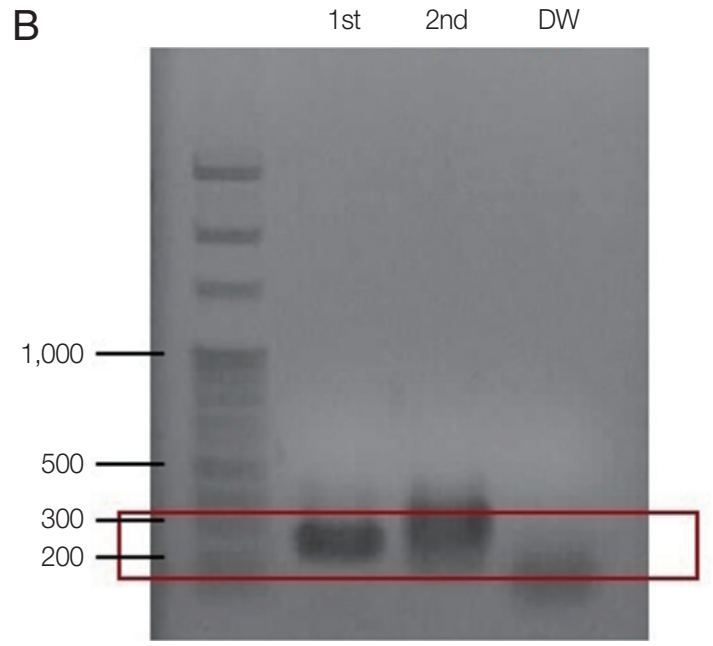

Fig. 3. Agarose gel-electrophoreses of babesiosis PCR amplicons for the second case. (A) PCR amplicon using Babesia- universal primers Bab 5 and Bab 8 (1st). For the nested PCR, primersBab 6 and Bab 7 were used (2nd) (B). PCR amplification using B. microtispecific primer pair (Bab 1 and Bab 4) producing 250 bp (1st) amplicon. Primer pair (Bab 2 and Bab 3) used for the 2nd amplification (2nd). DW, negative control.

range 12.0-15.3 g/dl), platelets 53,000 cells/ $\mu \mathrm{l}$ (normal range 150,000-400,000 cells/ $/ \mathrm{ll})$, erythrocyte sedimentation rate 69 $\mathrm{mm} / \mathrm{hr}$ (normal range 1-22 $\mathrm{mm} / \mathrm{hr}$ ), and C-reactive protein $9.52 \mathrm{mg} / \mathrm{dl}$ (normal range 0.0-0.3 mg/dl). Blood smear showed erythrocyte-containing organisms suspicious for Plasmodium species (Fig. 1). Abdomen-pelvis computed tomography revealed findings consistent with splenic infarction.

Suspecting malaria complicated with splenic infarction, treatment with hydroxychloroquine (800 mg loading and 400 mg 3 times after 6, 24, and $48 \mathrm{hr}$ ) was initiated. However, tests for detecting malaria antigens and polymerase chain reaction (PCR) for Plasmodium species failed to confirm malaria [16]. When the blood smear slide was re-examined, 4 Plasmodiumlike organisms in 1 erythrocyte emulating Maltese cross forms were observed (Fig. 2A). We prescribed atovaquone $250 \mathrm{mg}$ / proguanil hydrochloride $100 \mathrm{mg}$ and azithromycin $500 \mathrm{mg}$ to treat babesiosis. PCR for B. microti confirmed the diagnosis of babesiosis. A day after treatment, fever and abdominal discomfort subsided, and laboratory findings returned to normal.

\section{Case 2}

A 72-year-old woman without underlying disease visited our hospital for a fever that started 2 weeks before. She had been in Boston in the United States for 2 months previously. Vital signs were: blood pressure $93 / 67 \mathrm{mmHg}$, heart rate 76 beats per minute, respiratory rate 18 breaths per minute, and body temperature $37.7^{\circ} \mathrm{C}$. Laboratory findings showed white

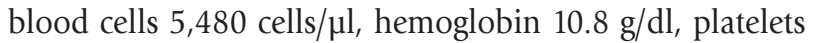
52,000 cells/ $/$ l, erythrocyte sedimentation rate $105 \mathrm{~mm} / \mathrm{hr}$, and C-reactive protein $15.1 \mathrm{mg} / \mathrm{dl}$. Malaria-like organisms were seen on the observation of blood smear; however, the malaria antigen test was negative. When the blood smear morphology was closely observed, Maltese cross forms were visible in the red blood cells; therefore, babesiosis was diagnosed (Fig. 1B). Atovaquone $250 \mathrm{mg} /$ proguanil $100 \mathrm{mg}$ and azithromycin $500 \mathrm{mg}$ were prescribed. However, fever persisted, and she developed pulmonary congestion. On diagnosis of severe babesiosis, we switched the medication to quinine and clindamycin, and red blood cell exchange was performed. As pulmonary congestion exacerbated, she was intubated due to respiratory failure (Fig. 2B). Doxycycline therapy was prescribed for possible coinfection with Borrelia or Anaplasma species.

PCR for B. microti was positive, confirming the diagnosis of babesiosis (Fig. 3). Also, the test for Borrelia burgdorferi was positive; IgG and IgM enzyme immunoassays titers were 1 : 256 and 1:128, respectively. Both IgG and IgM Western blots were also positive, confirming co-infection with Lyme disease. The patient expired in hospital on day 31 .

\section{Polymerase Chain Reaction for Babesia species}

For species identification, a nested PCR for B. microti was performed as described previously [17]. DNA was extracted from the whole blood of the patient using the QIAamp Blood Kit (Qiagen Co., Hilden, Germany). The first PCR was performed 
with the AccuPower PCR Premix (Bioneer Co., Daejeon, Korea), $2 \mu \mathrm{l}$ of purified genomic DNA, 10 pmol each of the forward (Bab 1; 5'-CTT AGT ATA AGC TTT TAT ACA GC-3') and reverse primers (Bab 4; 5'-ATA GGT CAG AAA CTT GAA TGA TAC A-3'), and the total volume was adjusted to $20 \mu \mathrm{l}$ with distilled water. Thermal cycling conditions were as follows: denaturing at $94^{\circ} \mathrm{C}$ for 5 min; 35 cycles of $40 \mathrm{sec}$ at $94^{\circ} \mathrm{C}, 40 \mathrm{sec}$ at $55^{\circ} \mathrm{C}$, and $40 \mathrm{sec}$ at $72^{\circ} \mathrm{C}$; and a final incubation at $72^{\circ} \mathrm{C}$ for $5 \mathrm{~min}$. A second PCR was performed using the same buffer and DNA polymerase enzyme. The first PCR product $(2 \mu \mathrm{l})$ was added and that different forward (Bab 2; 5'-GTT ATA GTT TAT TTG ATG TTC GTT T-3') and reverse (Bab 3; 5'-AAG CCA TGC GAT TCG CTA AT-3') primers were used. Theermal cycling conditions of the second PCR were as follows: denaturing at $94^{\circ} \mathrm{C}$ for $5 \mathrm{~min} ; 35$ cycles of $30 \mathrm{sec}$ at $94^{\circ} \mathrm{C}, 30 \mathrm{sec}$ at $55^{\circ} \mathrm{C}$, and $40 \mathrm{sec}$ at $72^{\circ} \mathrm{C}$; and a final incubation at $72^{\circ} \mathrm{C}$ for $5 \mathrm{~min}$. The PCR product was electrophoresed on a 1.5\%-agarose gel. Approximately 240 bp (exactly 238 bp) PCR product representing $B$. microti DNA was visualized using ultraviolet transillumination after staining with ethidium bromide.

\section{DISCUSSION}

Numerous mechanisms underlying Babesia infection have been documented, including blood transfusion $[18,19]$ and transplacental transmission $[20,21]$. However, infection with $B$. microti is most often observed following a tick bite in a person visiting or living in an area of endemicity. Although Babesia species are not endemic to Korea, 8 cases of infection have been reported: 1 endemic and 7 imported [5,7-11].

As the population of traveling abroad increases every year, both physicians and travelers need to be acquainted with not only endemic diseases of their provinces but also with those of other regions. Babesiosis is an increasingly imported disease in Korea [12].

The symptoms of babesiosis are easily mistaken for that of malaria. B. microti infection mimics malaria with symptoms of hemolytic anemia and a similar appearance of infected erythrocytes on blood smears. Moreover, malaria is endemic to the northern regions of South Korea. Therefore, malaria is easily assumed when patients in malaria-endemic regions present with high fever, malaise, and intra-erythrocyte parasites.

The patients we described here both resided in Incheon, a province where malaria is endemic. They were both initially mistaken as having malaria because of nonspecific febrile symptoms and intra-erythrocyte malaria-like protozoa on blood smears. However, negative results for the malaria antigen test and PCR and unresponsiveness to malaria treatment led to a suspicion of babesiosis. Minute history taking also aided the diagnosis. Both patients visited the northeastern part of the United States before the disease developed.

These 2 cases were also unique in that both patients presented unfamiliar complications of babesiosis. The patient of first case had splenic infarction, however, she did not have any hematologic or autoimmune disorders which may have caused embolic events. We suspect that she had splenic infarction as a complication of babesiosis. A few mechanisms underlying coagulopathy caused by babesiosis have been proposed, including microthrombus formation and local release of vasoactive factors by red blood cell lysis [22]. Additionally, in animal models, Babesia species-infected erythrocytes triggered an acute inflammatory response and activated the coagulation system, leading to increased erythrocyte adhesiveness to the capillaries [22].

The patient of second case was complicated by co-infection with Lyme disease. Ixodes ticks are known vectors in zoonosis and coinfection of tick-borne diseases such as Lyme disease, babesiosis, and human granulocytic anaplasmosis $[14,23]$. Particularly when patients do not improve clinically despite proper treatment, coinfection with other tick-borne diseases should be suspected. Babesiosis and Lyme disease are prevalent in overlapping regions of northeastern United States [14]. Moreover, epidemiologic studies have documented that approximately $2 / 3$ of patients with babesiosis experience concurrent Lyme disease [23].

In Korea, several imported cases of babesiosis and severe coinfection with malaria have been reported $[24,25]$. However, this is the first report of co-infection of B. microti and Lyme disease detected in Korea.

The reason that co-infection of tick-borne diseases causes fatality is not well-known. One theory is that once the immune system is weakened by babesiosis, the patient becomes vulnerable to other tick-borne protozoa infections [2,13]. It is also possible that a patient may be asymptomatic after exposure to B. microti when the immune system remains intact. When the immune system is, however, weakened by other tick-borne diseases, the patient may develop severe symptoms due to a synergy of the newly introduced infection and chronic asymptomatic babesiosis.

Our findings suggest that to diagnose tick-associated diseases, an easier method to detect B. microti and anaplasmosis 
should be devised in Korea. Not only is suspicion of babesiosis and other co-infected diseases crucial, but prompt confirmation of diagnoses is important to treat patients appropriately.

In conclusion, we reported 2 unique cases of babesiosis. One patient was complicated by splenic infarction, and the other was coinfected with Lyme disease. Although not endemic, physicians and travelers should be acquainted with babesiosis and its complications. Since coinfection with other tickborne diseases may aggravate the symptoms of babesiosis, easier and faster methods to detect Borrelia and Anaplasma species should be developed in Korea.

\section{ACKNOWLEDGEMENT}

This work was supported by a research grant from Inha University Hospital.

\section{REFERENCES}

1. Vannier E, Krause PJ. Update on babesiosis. Interdiscip Perspect Infect Dis 2009; 2009: 1-9.

2. Krause PJ. Babesiosis diagnosis and treatment. Vector Borne Zoonotic Dis 2003; 3: 45-51.

3. Usatii N, Khachatrian A, Stratidis J. Spontaneous splenic rupture due to Babesia microti infection: case report and review of the literature. IDCases 2014; 1: 63-65.

4. Tobler WD Jr, Cotton D, Lepore T, Agarwal S, Mahoney EJ. Case Report: successful non-operative management of spontaneous splenic rupture in a patient with babesiosis. World J Emerg Surg 2011; 6: 4 .

5. Yoon KT, Kim YA, Ku NS, Kim JH, Jung SJ, Kim HJ, Song KH, Choi YK, Shin SY, Kim YK, Kim MS, Park YS, Choi JY, Song YG, Kim JM. A Case of human babesiosis confirmed by polymerase chain reaction and treated with atovaquone and azithromycin. Infect Chemother 2006; 38: 300-303 (In Korean).

6. Na YJ, Chai JY, Jung BK, Lee HJ, Song JY, Je JY, Seo JH, Park SH, Choi JS, Kim MJ. An imported case of severe falciparum malaria with prolonged hemolytic anemia clinically mimicking a coinfection with babesiosis. Korean J Parasitol 2014; 52: 667-672.

7. Jang HJ, Kim JH, Park WJ, Kim CH, Kim DG, Hyun IG, Huh S, Kho WG, Chai JY. A Case of intraerythrocytic parasitism treated with quinine and clindamycin. Korean J Infect Dis 1998; 30: 478482 (In Korean).

8. Lee SS, Yang SY, Cho YK, Kim E, Kim YS, Woo JH, Ryu J, Chai JY. An imported case of babesiosis. Korean J Infect Dis 1997; 29: 4952.

9. Kweon SH, Kim YS, Pai H, Park J, Park H, Choi MH, Chai JY. A case of mixed infection with malaria and babesia. Korean J Infect
Dis 1998; 30: 198-202.

10. Sim JG, Park HS, Ahn DH, Kho WG, Chai JY. A case of babesiosis in an eight year old Korean boy. J Korean Pediatr Soc 1991; 34: 1417-1421 (In Korean).

11. Kim JY, Cho SH, Joo HN, Tsuji M, Cho SR, Park IJ, Chung GT, Ju JW, Cheun HI, Lee HW, Lee YH, Kim TS. First case of human babesiosis in Korea: detection and characterization of a novel type of Babesia sp. (KO1) similar to ovine Babesia. J Clin Microbiol 2007; 45: 2084-2087.

12. Ahn MH. Imported parasitic diseases in Korea. Infect Chemother 2010; 42: 271-279.

13. Krause PJ, McKay K, Gadbaw J, Christianson D, Closter L, Lepore T, Telford SR 3rd, Sikand V, Ryan R, Persing D, Radolf JD, Spielman A. Increasing health burden of human babesiosis in endemic sites. Am J Trop Med Hyg 2003; 63: 431-436.

14. Knapp KL, Rice NA. Human coinfection with Borrelia burgdorferi and Babesia microti in the United States. J Parasitol Res 2015; 2015: 1-11.

15. Moon S, Hong Y, Hwang KJ, Kim S, Eom J, Kwon D, Park JH, Youn SK, Sohn A. Epidemiological features and clinical manifestations of Lyme borreliosis in Korea during the period 2005-2012. Jpn J Infect Dis 2015; 68: 1-4.

16. Johnston SP, Pieniazek NJ, Xayavong MV, Slemenda SB, Wilkins PP, da Silva AJ. PCR as a confirmatory technique for laboratory diagnosis of malaria.. J Clin Microbiol 2006; 44: 1087-1089.

17. Persing DH, Mathiesen D, Marshall WF, Telford SR, Spielman A, Thomford JW, Conrad PA. Detection of Babesia microti by polymerase chain reaction. J Clin Microbiol 1992; 30: 2097-2103.

18. Jacoby GA, Hunt JV, Kosinski KS, Demirjian ZN, Huggins C, Etkind $\mathrm{P}$, Marcus LC, Spielman A. Treatment of transfusion-transmitted babesiosis by exchange transfusion. N Engl J Med 1980; 303: 1098-1100.

19. Perdrizet GA, Olson NH, Krause PJ, Banever GT, Spielman A, Cable RG. Babesiosis in a renal transplant recipient acquired through blood transfusion. Transplantation 2000; 70: 205-208.

20. New DL, Quinn JB, Qureshi MZ, Sigler SJ. Vertically transmitted babesiosis. J Pediatr 1997; 131: 163-164.

21. Esernio-Jenssen D, Scimeca PG, Benach JL, Tenenbaum MJ. Transplacental/perinatal babesiosis. J Pediatr 1987; 110: 570-572.

22. Goodger BV, Rode-Bramanis K, Wright IG. Procoagulant activity of Babesia bovis-infected erythrocytes. J Parasitol 1987; 73: 10521053.

23. Decicco A, Peiris BN, Kelly C, Latreille M, Jungkind D. Two cases of co-Infection with babesiosis and Lyme disease. Med Forum 2012; 13: 1-4.

24. Goo YK, Xuan X. New molecules in Babesia gibsoni and their application for diagnosis, vaccine development, and drug discovery. Korean J Parasitol 2014; 52: 345-354.

25. Zhou X, Li SG, Chen SB, Wang JZ, Xu B, Zhou HJ, Ge HX, Chen $\mathrm{JH}, \mathrm{Hu}$ W. Co-infections with Babesia microti and Plasmodium parasites along the China-Myanmar border. Infect Dis Poverty 2013; 2: 24 . 
